\title{
Co-infection of SARS-CoV-2 with Chlamydia or Mycoplasma pneumoniae: a case series and review of the literature
}

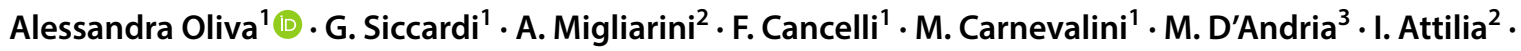 \\ V. C. Danese ${ }^{3} \cdot$ V. Cecchetti ${ }^{3} \cdot$ R. Romiti ${ }^{3} \cdot$ G. Ceccarelli ${ }^{1} \cdot$ C. M. Mastroianni ${ }^{1} \cdot$ P. Palange ${ }^{1} \cdot$ M. Venditti $^{1}$
}

Received: 20 June 2020 / Accepted: 15 July 2020

(c) The Author(s) 2020

\begin{abstract}
Introduction The novel coronavirus SARS-CoV-2 has spread all over the world causing a global pandemic and representing a great medical challenge. Nowadays, there is limited knowledge on the rate of co-infections with other respiratory pathogens, with viral co-infection being the most representative agents. Co-infection with Mycoplasma pneumoniae has been described both in adults and pediatrics whereas only two cases of Chlamydia pneumoniae have been reported in a large US study so far. Methods In the present report, we describe a series of seven patients where co-infection with $C$. pneumoniae $(n=5)$ or $M$. pneumoniae $(n=2)$ and SARS-CoV-2 was detected in a large teaching hospital in Rome.

Results and conclusion An extensive review of the updated literature regarding the co-infection between SARS-CoV-2 and these atypical pathogens is also performed.
\end{abstract}

Keywords SARS-CoV-2 infection · COVID-19 Mycoplasma pneumoniae $\cdot$ Chlamydia pneumoniae $\cdot$ Co-infection

\section{Introduction}

The novel coronavirus (now called SARS-CoV-2) initially discovered in Wuhan, China, has spread all over the world causing a global pandemic and representing a great medical challenge in terms of treatment, prevention and, not less important, diagnosis [1].

So far, there is limited knowledge on the rate of co-infections with other respiratory pathogens [2]. Likewise, data regarding bloodstream and respiratory bacterial and fungal

Electronic supplementary material The online version of this article (https://doi.org/10.1007/s15010-020-01483-8) contains supplementary material, which is available to authorized users.

Alessandra Oliva

alessandra.oliva@uniroma1.it

1 Department of Public Health and Infectious Diseases, Sapienza University of Rome, Piazzale Aldo Moro 5, 00185 Rome, Italy

2 Department of Translational and Precision Medicine, Sapienza University of Rome, Rome, Italy

3 Department of Clinical, Internal, Anestesiology and Cardiovascular Sciences, Sapienza University of Rome, Rome, Italy infections among patients with COVID-19 are very scarce and generally overlooked [3].

While early reports from China and Spain described co-infection as a rare event $(6 / 104,5.7 \%$ and $3 / 103,2.9 \%$, respectively) [4, 5], Kim et al. reported the presence of one or more additional pathogen in 24 of 116 patients (20.6\%) diagnosed with SARS-CoV-2 infection [6] whereas another study from China showed that up to $80 \%$ of SARS-CoV-2 infected subjects had IgM positivity against at least one respiratory agent, therefore highlighting how the detection of other respiratory pathogens cannot be used to rule out COVID-19 diagnosis [7]. Furthermore, it is still unknown whether co-infection with other pathogens, and in particular with intracellular atypical microorganisms, might play a role in determining the prognosis of SARS-CoV-2 infection.

In all the cases, viruses were the most representative agents [2-9]; on the other hand, scarce was the co-infection rate with Mycoplasma pneumoniae [10-16] and, interestingly, only two cases out of 1996 with Chlamydia pneumoniae has been described so far [17].

Herein, we describe patients where co-infection with Chlamydia pneumoniae or Mycoplasma pneumoniae and SARS-CoV-2 was detected in our teaching hospital in Rome, Italy. Furthermore, the updated literature regarding 
the co-infection between SARS-CoV-2 and these atypical pathogens is reviewed.

\section{Cases description}

We retrospectively analyzed data from clinical reports of all the patients admitted to Azienda Ospedaliero-Universitaria Policlinico Umberto I (Sapienza University) of Rome between 1 March and 30 April 2020 with documented SARS-CoV-2 infection. The study was approved by the local Ethics Committee (ID Prot. 109/2020). A total of 182 subjects were tested also for C. pneumoniae and $M$. pneumoniae. We found that seven patients (3.8\%) were coinfected with SARS-CoV-2 and atypical microorganisms (five C. pneumoniae, $2.7 \%$, two M. pneumoniae, $1.1 \%$ ). Diagnosis of $C$. pneumoniae and M. pneumoniae infection was made based on the serologies (DIESSE Diagnostica Senese S.p.A., sensitivity $97.4 \%$ and $94.7 \%$, specificity 94.1\% and $92.6 \%$ for C. pneumoniae and M. pneumoniae, respectively) [18] whereas SARS-CoV-2 diagnosis was based on nasopharyngeal swab positivity by using polymerase chain reaction (PCR) [19]. Definition of pneumonia or severe pneumonia was based on the WHO interim guidance and included clinical signs of pneumonia (fever, cough, dyspnoea, fast breathing) with or without signs of severe pneumonia such as respiratory rate $>30$ breaths/min, severe respiratory distress, or $\mathrm{SpO} 2<90 \%$ on room air $[19,20]$.

Clinical and laboratory characteristics of patients are listed in Table 1, radiological findings are shown in Supplementary Figure 1. Among the patients, four were male and three female, the median age was 73 years (IQR 45-79). All but one patient underwent CT-scan of the lungs, one patient underwent only chest X-ray, which showed bilateral interstitial involvement. Lung CT-scan showed multifocal, bilateral and prevalent peripheral infiltrates in six patients $(85.7 \%)$, ground glass in five patients $(71.4 \%)$, subpleural consolidation in four patients (57.1\%). No patient had pleural effusion. According to guidelines [19], severe pneumonia was observed in 2/7 (28.5\%) cases. All patients underwent therapy with hydroxychloroquine and azithromycin, 5/7 with heparin (57.1\%), 3/7 with corticosteroids (42.8\%), 2/7 with lopinavir/ritonavir (28.5\%), 2/7 with tocilizumab (28.5\%). One patient received also teicoplanin that has been described as potentially active against coronaviruses $[21,22]$. As for oxygen delivery, two patients $(28.5 \%)$ received high-oxygen non-invasive support (one high-flow nasal cannula, one C-PAP), three $(43.0 \%)$ were on Venturi masks and the remaining two $(28.5 \%)$ were on room air. All patients were discharged after a median length of hospitalization of 28 days (IQR 13-34).

Finally, when clinical outcomes (ICU admission and intra-hospital mortality) of 175 patients without $M$. pneumoniae or $C$. pneumoniae co-infection [median age 63 years (IQR 52-76), 71 (40.5\%) females] were compared to those with co-infection, no differences were observed [1/7 (14.2\%) vs. $24 / 175(13.7 \%)$ and $0 / 7$ (0\%) vs. $25 / 175$ (14.2\%), respectively].

\section{Discussion and review of the literature}

In the present report we described for the first time in Europe $[2,17]$ that patients with SARS-CoV-2 infection might be co-infected, among agents of atypical pneumonia, not only with $M$. pneumoniae but also with $C$. pneumoniae. These microorganisms can affect adults and children, are usually mild and only occasionally could represent life-threatening conditions. In particular, M. pneumoniae may cause epidemics and spread in close clusters. As the majority of symptomatic patients with SARS-CoV-2 infection develop an atypical pneumonia syndrome with fever, cough, and shortness of breath, co-infections with C. pneumoniae or M. pneumoniae are likely obscured, making therefore difficult the differential diagnosis only based on clinical presentation [19, 20]. The rate of co-infection with M. pneumoniae in SARS$\mathrm{CoV}-2$ pneumonia patients has been reported in the literature [10-16] whereas co-infection with $C$. pneumoniae has been reported only in two cases in a large US study involving 5700 patients with COVID-19 [17] (Table 2). In detail, Fan et al. described a case of a 36-year old male requiring Intensive Care Unit (ICU) admission and presenting with severe lymphopenia, low platelet count and cold agglutinin titer of 1:8 with M. pneumoniae antibody titer of 1:160 [14] whereas Ziang Gao et al. described a case of 49-year old female presenting with cough, expectoration and lung CT scan showing multiple ground-glass opacities in bilateral lower lobes [16]. Gayam et al. reported that six out of 350 patients $(1.71 \%)$ with SARS-CoV-2 infection were also diagnosed with $M$. pneumoniae detected by serology [12] and, in a recent double-center Chinese study conducted at Qingdao and Wuhan regions and involving 68 patients with SARS-CoV-2 infection, the authors found a not-negligible rate of co-infection with common respiratory pathogens, with $8 / 68$ (11.7\%) of subjects showing also M. pneumoniae positive serology [7]. In the same study, a highly different distribution between the two regions (7/30, 23.3\%, in Qingdao and 1/38, 2.63\%, in Wuhan) was observed [7]. Although the whole rate of coinfection was far different, retrospective studies conducted in Spain and in the UK showed a similar number of SARSCoV-2-M. pneumoniae co-infection $(0.97 \%$ and $1.49 \%$, respectively), the latter detected with multiplex PCR assays $[5,11]$. In pediatric patients, co-infection with $M$. pneumoniae was surprisingly high, accounting for $16 / 34(47.0 \%)$ of the total and a case report described the presence of COVID19 infection with pleural effusion complicated by secondary 


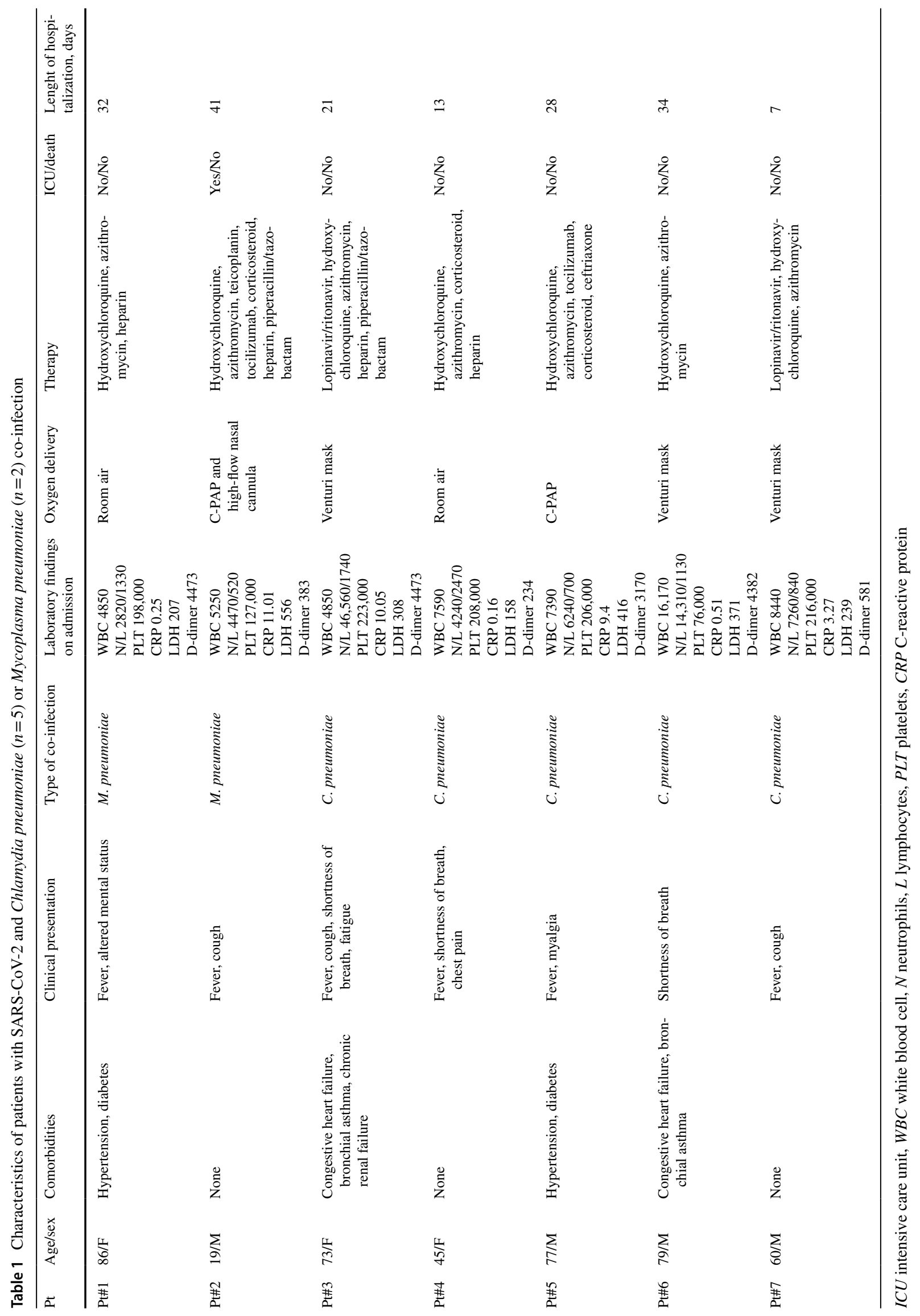




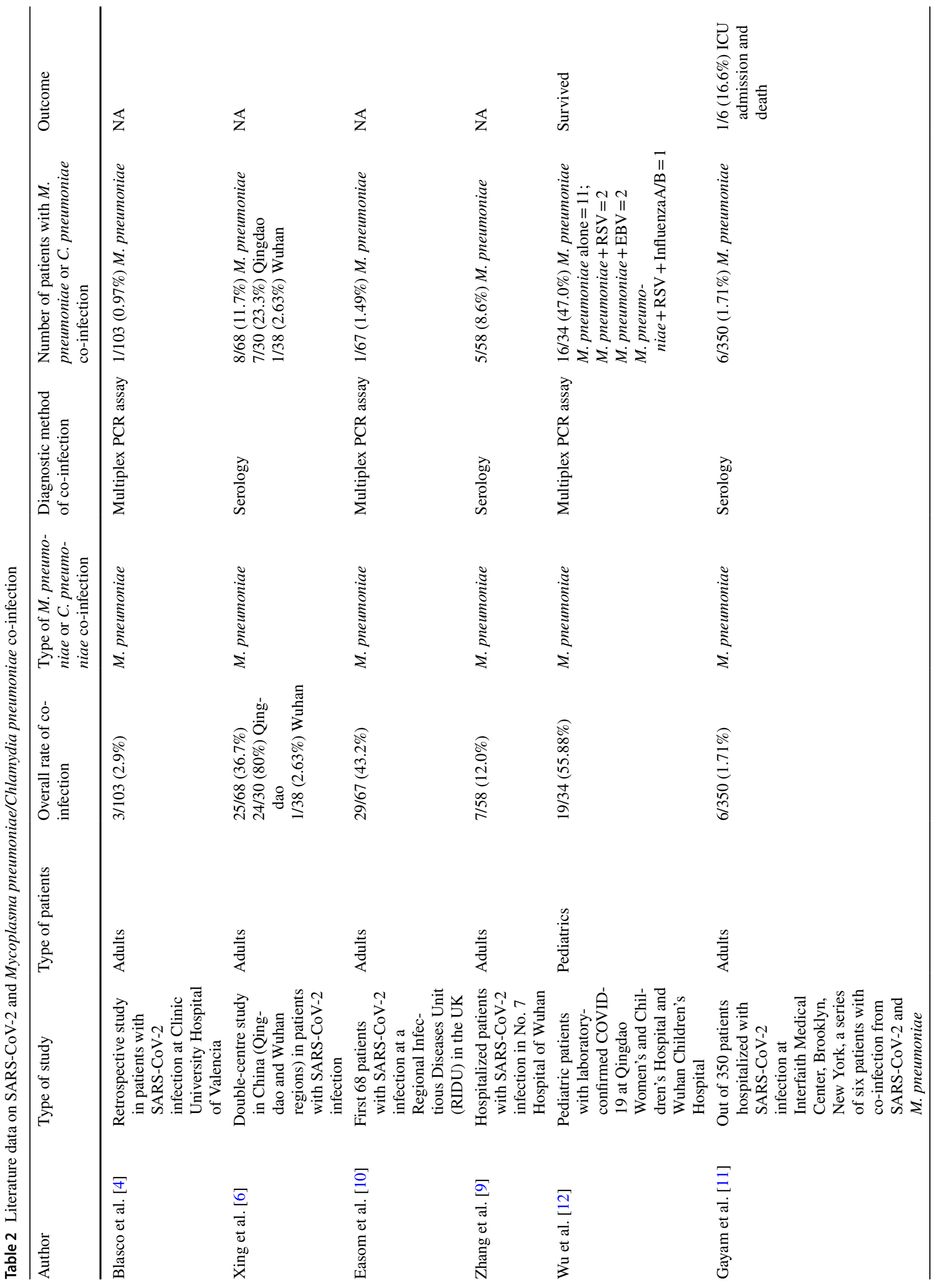




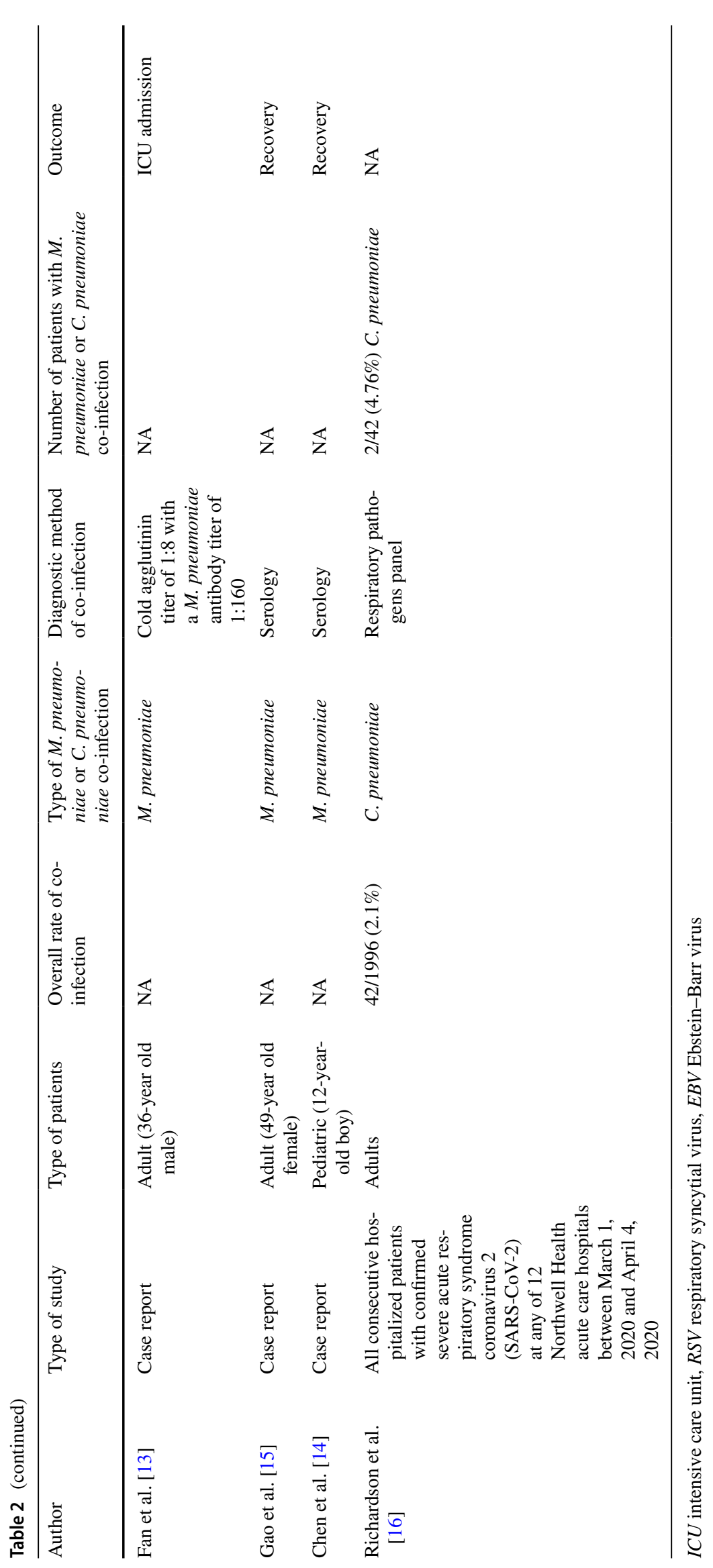


M. pneumoniae infection in a 12-year old boy $[13,15]$. As for $C$. pneumoniae, only one large US study which had the aim to describe the clinical characteristics and outcomes of 5700 hospitalized patients with COVID-19 found two $C$. pneumoniae cases out of 42/1996 positive samples tested also for respiratory pathogens panel [17]. Of note, and unlike our report, no clinical information of these two cases of $C$. pneumoniae and SARS-CoV-2 co-infection were available [17].

Similarly to what has been reported in the literature, the majority of our patients presented with fever, cough and/or shortness of breath, showed bilateral infiltrates at the lung $\mathrm{CT}$, received oxygen support and were treated with hydroxychloroquine and azithromycin.

The possible co-existence of pathogens other than SARSCoV-2 in patients with COVID-19 infection focuses the attention on the real incidence of SARS-CoV-2 and other bacterial/viral or even fungal co-infections, which should be investigated to find whether co-infections might play a role in disease severity and/or mortality [2]. In our case series, only one patient needed ICU admission, no patients died and the median duration of hospitalization was 28 days.

The present report has several limitations. First, not all the hospitalized patients with SARS-CoV-2 infection were tested also for $C$. pneumoniae and $M$. pneumoniae; therefore, we could present only a part of patients with serological detection of atypical pathogens and infection with SARS$\mathrm{CoV}-2$ and the real incidence of co-infection cannot be truly established, requiring the need of testing always for pathogens other than SARS-CoV-2. Then, for the diagnosis of co-infections we could rely only on serology, since molecular analyses of respiratory samples specifically detecting M. pneumoniae or C. pneumoniae were lacking. In fact, although rarely, serology might be limited by possible false positive results, which should always be taken into account when deciding to exclude SARS-CoV-2 infection. One additional limitation is represented by the lack of paired samples to confirm prior serological results for the diagnosis of atypical pathogens.

However, with these limitations in mind, we reported for the first time the clinical characteristics of patients with $C$. pneumoniae, and not only M. pneumoniae, as a co-existing pathogen during SARS-CoV-2 infection. Therefore, the present report opens the path to additional studies investigating the real incidence of co-infections during SARSCoV-2 epidemic and their possible impact on infection severity and mortality. Not less important, keeping in mind that in the future SARS-CoV-2 might be sporadic and not the cause of a pandemic infection anymore, we could infer that the serological detection of these atypical pulmonary pathogens in subjects presenting with respiratory symptoms cannot be used to rule out a diagnosis of COVID-19 [2, 4, $7,23]$. On the other hand, the reliability of serology for atypical bacteria should be considered when excluding the diagnosis of COVID-19 in patients with nasopharyngeal negative swabs (which has been demonstrated to occur in a not-negligible percentage of cases) [24], symptoms highly suggestive of SARS-CoV-2 infection and positive serology for other pathogens. Based on these considerations, physicians should assume that the presence of a pathogen other than SARS-CoV-2 does not ensure that a subject does not have also COVID-19.

In conclusion, SARS-CoV-2 infection might be associated with other common respiratory pathogens, including those causing atypical pneumonia. This finding should be considered in the near future, especially when ruling out the diagnosis of COVID-19. Therefore, the search for SARSCoV-2 infection should be added to routine diagnostic testing even though other common respiratory pathogens are detected. Further studies are needed to evaluate the possible influence of co-infections on the severity of SARS-CoV-2 infection.

\section{Data availability statement}

The data used to support the findings of this study are available from the corresponding author upon request.

Acknowledgements Open access funding provided by Università degli Studi di Roma La Sapienza within the CRUI-CARE Agreement. The authors thank Roberta Pisano for performing Chlamydia and Mycoplasma pneumoniae serology and the nursing staff for their contribution to sample collection.

Funding This research did not receive specific grants or funding.

\section{Compliance with ethical standards}

Conflict of interest The authors declare no financial and non-financial competing interests.

Open Access This article is licensed under a Creative Commons Attribution 4.0 International License, which permits use, sharing, adaptation, distribution and reproduction in any medium or format, as long as you give appropriate credit to the original author(s) and the source, provide a link to the Creative Commons licence, and indicate if changes were made. The images or other third party material in this article are included in the article's Creative Commons licence, unless indicated otherwise in a credit line to the material. If material is not included in the article's Creative Commons licence and your intended use is not permitted by statutory regulation or exceeds the permitted use, you will need to obtain permission directly from the copyright holder. To view a copy of this licence, visit http://creativecommons.org/licenses/by/4.0/.

\section{References}

1. Country and technical guidance-coronavirus disease (COVID19) [website]. Geneva: World Health Organization; 2020. https 
://www.who.int/emergencies/diseases/novel-coronavirus-2019/ technical-guidance.

2. Lai C-C, Wang C-Y, Hsueh P-R. Co-infections among patients with COVID-19: the need for combination therapy with nonanti-SARS-CoV-2 agents?. J Microbiol Immunol Infect. 2020. https://doi.org/10.1016/j.jmii.2020.05.013.

3. Antinori S, Galimberti L, Milazzo L, Ridolfo AL. Bacterial and fungal infections among patients with SARS-CoV-2 pneumonia. Infez Med Suppl. 2020;1:29-36.

4. Wang M, Wu Q, Xu W, et al. Clinical diagnosis of 8274 samples with 2019-novel coronavirus in Wuhan. MedRxiv Prepr. 2020. https://doi.org/10.1101/2020.02.12.20022327[Posted online February 18, 2020].

5. Blasco ML, Buesa J, Colomina J, et al. Co-detection of respiratory pathogens in patients hospitalized with coronavirus viral disease-2019 pneumonia. J Med Virol. 2020. https://doi. org/10.1002/jmv.25922.

6. Kim D, Quinn J, Pinsky B, et al. Rates of co-infection between SARS-CoV-2 and other respiratory pathogens. JAMA. 2020;323(20):2085-2086. https://doi.org/10.1001/ jama.2020.6266.

7. Xing Q, Li G, Xing Y, et al. Precautions are needed for COVID19 patients with coinfection of common respiratory pathogens. MedRxiv Prepr. 2020. https://doi.org/10.1101/2020.02.29.20027 698[Posted online March 5, 2020].

8. Nowak MD, Sordillo EM, Gitman MR, Paniz Mondolfi AE. Coinfection in SARS-CoV-2 infected patients: where are influenza virus and rhinovirus/enterovirus? J Med Virol. 2020. https://doi. org/10.1002/jmv.25953.10.1002/jmv.25953.

9. Wee LE, Ko KKK, Ho WQ, et al. Community-acquired viral respiratory infections amongst hospitalized inpatients during a COVID-19 outbreak in Singapore: co-infection and clinical outcomes. J Clin Virol. 2020;128:104436. https://doi.org/10.1016/j. jcv.2020.104436.

10. Zhang J-J, Dong X, Cao Y-Y, et al. Clinical characteristics of 140 patients infected with SARS-CoV-2 in Wuhan, China. Allergy. 2020. https://doi.org/10.1111/all.14238[Online ahead of print].

11. Easom N, Moss P, Barlow G, et al. Sixty-eight consecutive patients assessed for COVID-19 infection: experience from a UK regional infectious diseases unit. Influenza Other Respir Viruses. 2020. https://doi.org/10.1111/irv.12739[Online ahead of print].

12. Gayam V, Konala VM, Naramala S, et al. Presenting characteristics, comorbidities, and outcomes of patients coinfected with COVID-19 and mycoplasma pneumoniae in the USA. J Med Virol. 2020. https://doi.org/10.1002/jmv.26026[Online ahead of print].
13. Wu Q, Xing Y, Shi L, et al. Coinfection and other clinical characteristics of COVID-19 in children. Pediatrics. 2020. https:// doi.org/10.1542/peds.2020-0961[Online ahead of print].

14. Fan BE, Lim K, Lian Chong VC, et al. COVID-19 and mycoplasma pneumoniae coinfection. Am J Hematol. 2020;95:723-4. https://doi.org/10.1002/ajh.25785[Epub 2020 Apr 3].

15. Chen H-R, Zou H, Xue M, et al. A case of childhood COVID19 infection with pleural effusion complicated by possible secondary mycoplasma pneumoniae infection. Pediatr Infect Dis J. 2020;39:e135-e137137. https://doi.org/10.1097/INF.00000 00000002721.

16. Gao Z, Gao L, Chen X, Xu Y. A 49-year-old woman co-infected with SARS-COV-2 and mycoplasma-a case report. Res Sq Prepr. 2020. https://doi.org/10.21203/rs.3.rs-16376/v1[Posted online 08 Mar, 2020].

17. Richardson S, Hirsch JS, Narasimhan M, et al. Presenting characteristics, comorbidities, and outcomes among 5700 patients hospitalized with COVID-19 in the New York City area. JAMA. 2020;323:2052-9. https://doi.org/10.1001/ jama.2020.6775[Online ahead of print].

18. https://www.diesse.it/.

19. World Health Organization. Clinical management of COVID-19, interim guidance 27 May 2020. https://www.who.int/publicatio ns/i/item/clinical-management-of-covid-19.

20. IMAI District Clinician Manual. Hospital care for adolescents and adults. Geneva: World Health Organization; 2020. https:// apps.who.int/iris/bitstream/handle/10665/77751/9789241548 290_Vol2_eng.pdf?sequence. Accessed 13 May 2020.

21. Ceccarelli G, Alessandri F, d'Ettorre G, et al. Is teicoplanin a complementary treatment option for COVID-19? The question remains. Int J Antimicrob Agents. 2020. https://doi. org/10.1016/j.ijantimicag.2020.106029[Online ahead of print].

22. Baron SA, Devaux C, Colson P, et al. Teicoplanin: an alternative drug for the treatment of COVID-19? Int J Antimicrob Agents. 2020;55:105944. https://doi.org/10.1016/j.ijantimica g.2020.105944[Epub 2020 Mar 13].

23. Zahariadis G, Gooley TA, Ryall P, et al. Risk of ruling out severe acute respiratory syndrome by ruling in another diagnosis: variable incidence of atypical bacteria coinfection based on diagnostic assays. Can Respir J. 2006;13:17-22. https://doi. org/10.1155/2006/862797.

24. Wang W, Xu Y, Gao R, et al. Detection of SARS-CoV-2 in different types of clinical specimens. JAMA. 2020;323:1843-4. https ://doi.org/10.1001/jama.2020.3786 [Online ahead of print]. 